\title{
Precision Electroweak Measurements in ATLAS and CMS
}

\author{
Daniele Zanzi* on behalf of the ATLAS and CMS Collaborations \\ CERN \\ E-mail: daniele.zanzi@cern.ch
}

This talk gives an overview of the measurements of the effective leptonic weak mixing angle $\sin ^{2} \theta_{\text {eff }}^{\ell}$ and of the $W$ boson mass $m_{W}$ with the ATLAS and CMS experiments at the LHC, with a focus on dominant theoretical uncertainties and on prospects for future measurements. It also presents results from recent $W \rightarrow \ell v$ and $Z \rightarrow \ell \ell$ cross section measurements, which are important inputs for the $\sin ^{2} \theta_{\text {eff }}^{\ell}$ and $m_{W}$ measurements.

7th Annual Conference on Large Hadron Collider Physics - LHCP2019

20-25 May, 2019

Puebla, Mexico

${ }^{*}$ Speaker. 


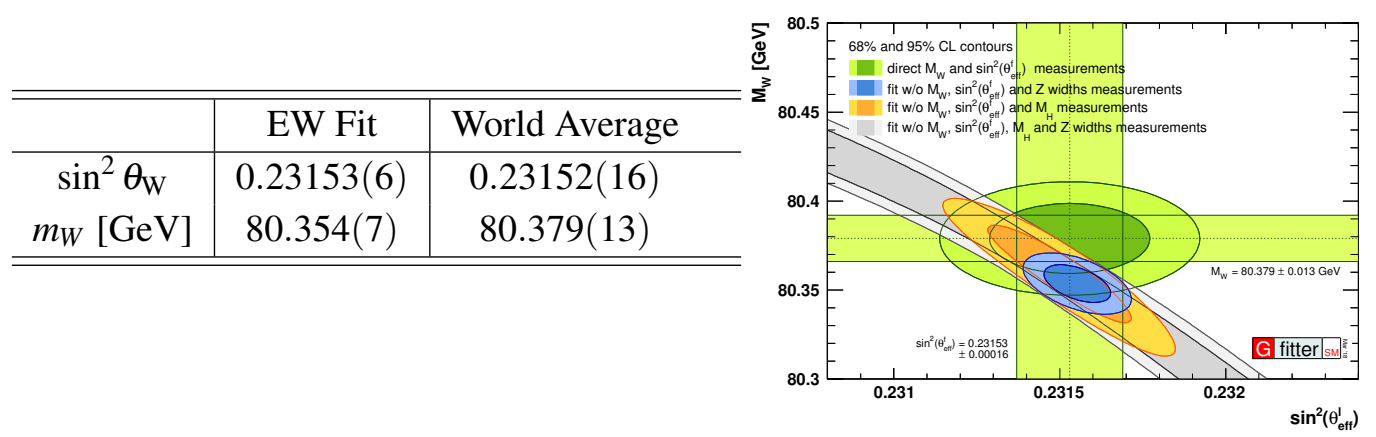

Figure 1: Comparison of measured and predicted values of $\sin ^{2} \theta_{\mathrm{eff}}^{\ell}$ and $m_{W}$. The measured values are the world averages of the direct measurements, while the predicted values are indirectly determined by the global fit of the Standard Model electroweak sector without the observable of interest as input [1].

\section{Introduction}

The precise measurement of the parameters of the Standard Model (SM) is a pillar of the physics programme of the Large Hadron Collider (LHC). Parameters of the SM, like the weak mixing angle $\sin ^{2} \theta_{\mathrm{W}}$ and the $W$ boson mass $m_{W}$, can be directly measured, but also predicted within the SM from measurements of other parameters, like the $Z$ boson mass, the electromagnetic coupling constant and the Fermi constant [1]. The comparison of of measured and predicted values of $\sin ^{2} \theta_{\mathrm{W}}$ and $m_{W}$ is a crucial test of the SM, but to be significant it requires measurements as precise as predictions. The experimental goal is to measure $m_{W}$ and $\sin ^{2} \theta_{\mathrm{eff}}^{\ell}$ at a precision of about $10 \mathrm{MeV}$ and $10^{-4}$, respectively (Fig. 1), where $\sin ^{2} \theta_{\text {eff }}^{\ell}$ is the effective leptonic weak mixing angle, defined as the weak mixing angle times a flavour-dependent form factor that includes electroweak higher-order corrections.

Experiments at the LHC are reaching the highest precision for single-experiment measurements both for $\sin ^{2} \theta_{\text {eff }}^{\ell}$ and for $m_{W}$ with the $p p$ collision data collected at $\sqrt{s}=7$ and $8 \mathrm{TeV}$, during the LHC Run 1. Analyses of larger datasets collected during the Run 2 or to be collected in future LHC data-taking periods will provide even more precise measurements by improving on statistical and experimental uncertainties.

The bottleneck in the precision of these measurements at the LHC are the theoretical uncertainties in the interpretation of the measured data in terms of $\sin ^{2} \theta_{\text {eff }}^{\ell}$ and $m_{W}$. The dominant uncertainty comes from our limited knowledge of the proton structure. To address this limitation, the LHC experiments are providing a wide range of high-precision measurements that can be used to improve predictions and reduce the theoretical uncertainties of the $\sin ^{2} \theta_{\mathrm{eff}}^{\ell}$ and $m_{W}$ measurements.

This talk gives an overview of the $\sin ^{2} \theta_{\text {eff }}^{\ell}$ and $m_{W}$ measurements performed by the ATLAS [2] and CMS [3] experiments at the LHC, with a focus on the main theoretical uncertainties and on prospects for future measurements. It also presents results from recent $W \rightarrow \ell v$ and $Z \rightarrow \ell \ell$ cross section measurements, where $\ell=e, \mu$.

\section{Measurement of the $W$ boson mass}

The ATLAS experiment measured the $W$ boson mass at a value of $m_{W}=80370 \pm 19 \mathrm{MeV}$ in 


\begin{tabular}{l|r}
\hline \hline Value [MeV] & 80369.5 \\
\hline Statistical Uncertainty & \pm 6.8 \\
Muon Uncertainty & \pm 6.6 \\
Electron Uncertainty & \pm 6.4 \\
Recoil Uncertainty & \pm 2.9 \\
Background Uncertainty & \pm 4.5 \\
QCD Uncertainty & \pm 8.3 \\
EW Uncertainty & \pm 5.5 \\
PDF Uncertainty & \pm 9.2 \\
\hline Total Uncertainty & \pm 18.5 \\
\hline \hline
\end{tabular}

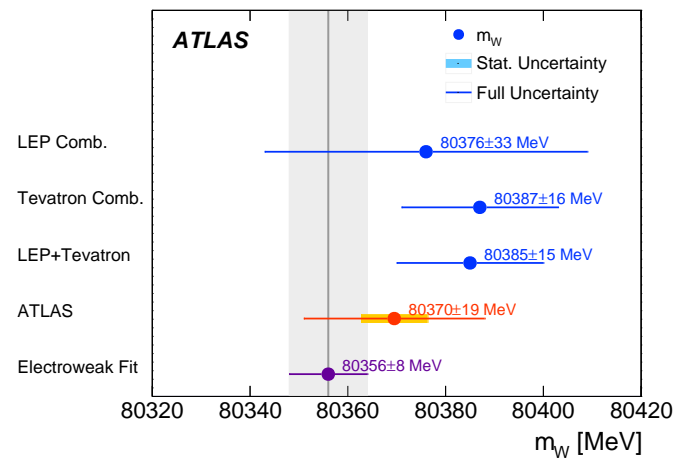

Figure 2: Breakdown of the uncertainty on the $m_{W}$ measurement performed by ATLAS (left) and comparison of the ATLAS measurement with combined measurements performed by experiments at LEP and Tevatron, as well as with the prediction from the electroweak fit (right) [4].

$W \rightarrow \ell v$ events with $4.6 \mathrm{fb}^{-1}$ of $p p$ data collected at $\sqrt{s}=7 \mathrm{TeV}$ [4].

The measurement is based on a template fit to the distributions of the charged lepton transverse momentum $p_{\mathrm{T}}^{\ell}$ and the transverse mass of the $W$ boson candidate $m_{\mathrm{T}}=\sqrt{2 p_{\mathrm{T}}^{\ell} p_{\mathrm{T}}^{\text {miss }}(1-\cos \Delta \phi)}$, where $\vec{p}_{\mathrm{T}}^{\text {miss }}=-\left(\vec{p}_{\mathrm{T}}^{\ell}+\vec{u}_{\mathrm{T}}\right)$ is the missing transverse momentum and $\Delta \phi$ is the azimuthal opening angle between the charged lepton and the missing transverse momentum. The recoil in the transverse plane $\vec{u}_{\mathrm{T}}$ is reconstructed from the vector sum of the transverse energy of all clusters reconstructed in the calorimeters, excluding energy deposits associated with the charged lepton.

The fit to the $p_{\mathrm{T}}^{\ell}$ and $m_{\mathrm{T}}$ distributions improves the precision of the measurement compared to the precision of the fits to individual distributions since these observables are affected by different modelling uncertainties. The $p_{\mathrm{T}}^{\ell}$ distribution is sensitive to the modelling of the transverse momentum of the $W$ boson $\left(p_{\mathrm{T}}^{W}\right)$, while the $m_{\mathrm{T}}$ distribution is primarily determined by the reconstructed $\vec{u}_{\mathrm{T}}$ which is sensitive to the modelling of effects like underlying event, multi-parton interactions and additional simultaneous $p p$ collisions. The templates used in the fit are built using the Powheg MC generator [5] interfaced with PyTHIA 8 [6], and reweighted using predictions from DYNNLO [7] and PYTHIA 8 with the AZ tune [8] to improve the modelling of the $W$ boson's rapidity, transverse momentum and polarisation.

As shown in Fig. 2, the ATLAS result is almost as precise as the combination of the measurements performed by experiments at LEP and Tevatron [4]. Its precision is mostly driven by uncertainties on the description of the proton structure with the Parton Density Functions (PDF) and on other QCD effects.

PDF uncertainties affect the $m_{W}$ measurement as they determine flavour and momentum of the incoming partons, which then impact the $W$ boson's rapidity, transverse momentum and polarisation. In future measurements, these uncertainties can be tackled with improved PDF sets based on fits to more measurements of $W$ and $Z$ boson differential cross sections at LHC, like those presented in Sec. 4, and possibly also more precise predictions, as discussed in Ref. [9].

QCD uncertainties are dominated by uncertainties on the $p_{\mathrm{T}}^{W}$ modelling. To improve on the limited precision of the $p_{\mathrm{T}}^{W}$ predictions, the $p_{\mathrm{T}}^{W}$ spectrum is determined from data. The precision of the direct measurement of $p_{\mathrm{T}}^{W}$ is driven by the resolution on the hadronic recoil $\sigma\left(u_{\mathrm{T}}\right)$, which 

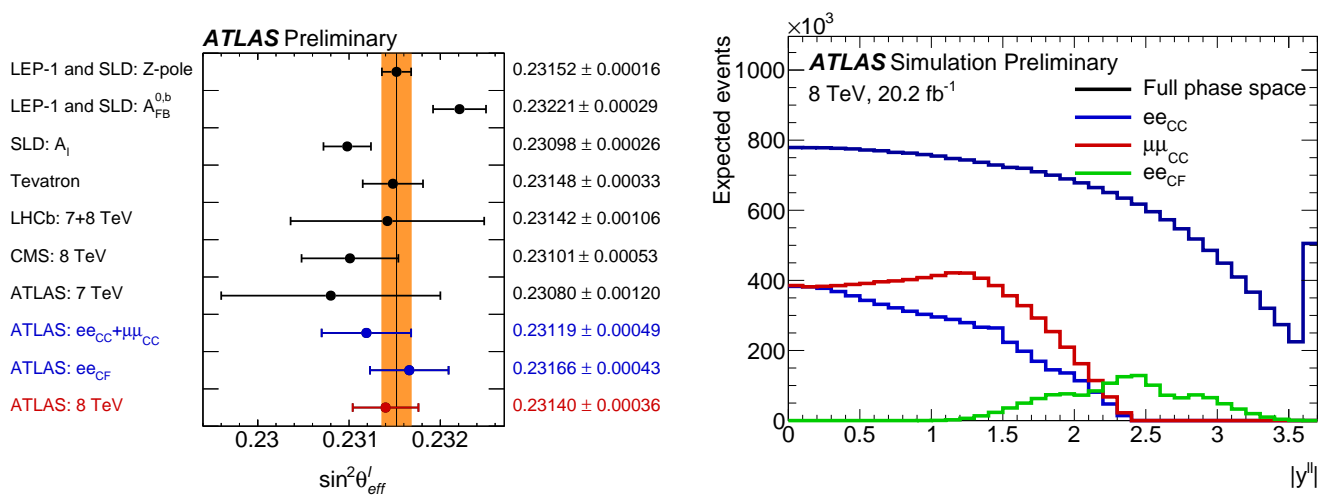

Figure 3: Left: summary of $\sin ^{2} \theta_{\text {eff }}^{\ell}$ measurements at the LEP, SLD, Tevatron and LHC colliders. The results of the ATLAS measurement at $\sqrt{s}=8 \mathrm{TeV}$ are presented also for the individual channels. Right: expected $Z$ boson signal event yields as a function of $\left|y^{\ell \ell}\right|$ in the full phase space of the decay leptons and in the $e e_{\mathrm{CC}}, \mu \mu_{\mathrm{CC}}$ and $e e_{\mathrm{CF}}$ analysis channels in the ATLAS measurement at $\sqrt{s}=8 \mathrm{TeV}$ [13].

strongly increases with the number of simultaneous $p p$ collisions (pile-up, $\langle\mu\rangle$ ). At $\langle\mu\rangle \approx 9$, the $W \rightarrow \ell v$ events at $\sqrt{s}=7 \mathrm{TeV}$ would not allow for a precise $p_{\mathrm{T}}^{W}$ measurement. Therefore, the $p_{\mathrm{T}}^{W}$ spectrum is determined by extrapolating $Z \rightarrow \ell \ell$ data using PYTHIA 8 predictions "tuned" to the measured $p_{\mathrm{T}}^{\ell \ell}[8]$. This procedure yields an uncertainty on $p_{\mathrm{T}}^{W}$ of $2.5 \%$ at low $p_{\mathrm{T}}^{W}$, mostly dominated by uncertainties on the modelling of the differences in the initial states of the $W$ and $Z$ boson productions [10]. The goal of future measurements is to halve the QCD uncertainties by directly measuring the $p_{\mathrm{T}}^{W}$ spectrum at $1 \%$ precision in the range $p_{\mathrm{T}}^{W}<5 \mathrm{GeV}$ using $W \rightarrow \ell v$ events collected at $\langle\mu\rangle \approx 2$. As described in Ref. [10], in data at such low pile-up, with lower calorimeter thresholds, and with an improved particle-flow $\vec{u}_{\mathrm{T}}$ reconstruction algorithm [11], the resolution on the hadronic recoil is expected to be $\sigma\left(u_{\mathrm{T}}\right)<5 \mathrm{GeV}$. ATLAS and CMS collected about $380 \mathrm{pb}^{-1}$ and $260 \mathrm{pb}^{-1}$ at $\sqrt{s}=13 \mathrm{TeV}$, and $200 \mathrm{pb}^{-1}$ and $300 \mathrm{pb}^{-1}$ at $\sqrt{s}=5 \mathrm{TeV}$ of data, respectively, both at $\langle\mu\rangle \approx 2$, and such data are expected to provide enough $W$ boson candidates to reach the $\sigma\left(p_{\mathrm{T}}^{W}\right) \sim 1 \%$ target.

\section{Measurement of the effective leptonic weak mixing angle}

The combined average of the most precise six measurements of the effective leptonic weak mixing angle from the LEP and SLD colliders yields a value of $\sin ^{2} \theta_{\text {eff }}^{\ell}=0.23153 \pm 0.00016$ [12], with a 3.2 standard-deviation difference between the two most precise individual measurements

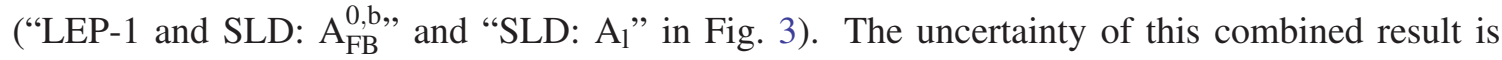
twice as larger as the uncertainty on the prediction for $\sin ^{2} \theta_{\text {eff }}^{\ell}$ from the global electroweak fit [1]. Measurements at the LHC are so far less precise than the combination of measurements at the LEP, SLD and Tevatron colliders, though the preliminary ATLAS result of $\sin ^{2} \theta_{\text {eff }}^{\ell}=0.23140 \pm 0.00036$ [13] is almost as precise as the individual LEP, SLD and Tevatron measurements (Fig. 3).

At the LHC, $\sin ^{2} \theta_{\text {eff }}^{\ell}$ is measured from the asymmetry in the angular distributions of the leptons from $Z \rightarrow \ell \ell$ decays. The ATLAS and CMS experiments measured $\sin ^{2} \theta_{\text {eff }}^{\ell}$ with $20.2 \mathrm{fb}^{-1}$ and up to $19.6 \mathrm{fb}^{-1}$ of $p p$ data collected at $\sqrt{s}=8 \mathrm{TeV}$, respectively, with two different approaches. 
In the ATLAS measurement [13], $\sin ^{2} \theta_{\mathrm{eff}}^{\ell}$ is inferred from the measurement of the $A_{4}$ angular coefficient, one of eight coefficients which, together with the unpolarised cross section, describe entirely the production dynamics of the $Z$ boson in perturbative $\mathrm{QCD}$. The angular coefficients are measured in the full phase space of the decay leptons from the reconstructed angular distributions of the leptons in the Collins-Soper frame [14]. From the $A_{4}, \sin ^{2} \theta_{\text {eff }}^{\ell}$ is derived using an effective linear relation which includes higher-order electroweak corrections. While the measurement of $A_{4}$ is dominated by statistical uncertainties, the precision of the interpretation of $A_{4}$ in terms of $\sin ^{2} \theta_{\text {eff }}^{\ell}$ is dominated by QCD and PDF uncertainties.

The measurement of $\sin ^{2} \theta_{\mathrm{eff}}^{\ell}$ by CMS [15] is performed based on the forward-backward asymmetry $A_{\mathrm{FB}}=\left(\sigma\left(\cos \theta^{*}>0\right)-\sigma\left(\cos \theta^{*}<0\right)\right) /\left(\sigma\left(\cos \theta^{*}>0\right)+\sigma\left(\cos \theta^{*}<0\right)\right)$, where $\theta^{*}$ is the polar angle of the negative lepton in the Collins-Soper frame, in the fiducial phase space. A fit to the observed $A_{\mathrm{FB}}$ is performed with templates built from predictions with varying $\sin ^{2} \theta_{\text {eff }}^{\ell}$ values. Like the ATLAS measurement, the CMS measurement is dominated by QCD and PDF uncertainties.

At the LHC, the magnitudes of the reconstructed $A_{4}$ and $A_{\mathrm{FB}}$ asymmetries depend primarily on the momentum of the initial state quarks. In the LHC $p p$ collisions, the initial state valence quark is assigned based on the rapidity of the reconstructed $Z$ boson, as the valence quark tends to carry a larger fraction of the proton momentum than the sea quark. In forward events with large $\left|y^{\ell \ell}\right|$, this assignment is more accurate than in central events with low $\left|y^{\ell \ell}\right|$ and the magnitudes of the reconstructed $A_{4}$ and $A_{\mathrm{FB}}$ are larger. Due to such dilution, events at large $\left|y^{\ell \ell}\right|$ have a larger statistical power compared to those at small $\left|y^{\ell \ell}\right|$. As shown in Fig. 3, in the ATLAS measurement the $e e_{\mathrm{CF}}$ channel, which selects events with one central electron $\left(\left|\eta^{\ell}\right|<2.4\right)$ and one forward electron $\left(2.5<\left|\eta^{\ell}\right|<4.9\right)$, reaches $\left|y^{\ell \ell}\right|$ values significantly higher than the central channels with two central electrons $\left(e e_{\mathrm{CC}}\right)$ or muons $\left(\mu \mu_{\mathrm{CC}}\right)$. Thanks to this, even with a much smaller signal event yield, the precision in $e e_{\mathrm{CF}}$ is better than the precision in $e e_{\mathrm{CC}}+\mu \mu_{\mathrm{CC}}$ combined. The CMS measurement is performed in the central channels at the same precision of the central channels in the ATLAS result.

Both the ATLAS result of $\sin ^{2} \theta_{\text {eff }}^{\ell}=0.23140 \pm 0.00021$ (stat) $\pm 0.00024(\mathrm{PDF}) \pm 0.00016$ (syst) and the CMS result of $\sin ^{2} \theta_{\text {eff }}^{\ell}=0.23101 \pm 0.00036$ (stat) \pm 0.00031 (PDF) \pm 0.00024 (syst) are dominated by uncertainties on the PDF modelling, as this determines the momentum of the initial state quarks, hence the magnitude of the reconstructed $A_{4}$ and $A_{\mathrm{FB}}$. To reduce the impact of the PDF uncertainties, correlations in the expected $A_{4}$ and $A_{\mathrm{FB}}$ distributions as functions of $\left|y^{\ell \ell}\right|$ and $m^{\ell \ell}$ induced by PDF variations are used to constrain the PDF uncertainties in-situ to data. In the ATLAS measurement, the PDF uncertainties are "profiled" to the parameters of interest of the fit, while in the CMS measurement a Bayesian $\chi^{2}$ reweighting is used. These methods yield significant reductions in the size of the PDF uncertainties, a factor of 2 in reduction in the CMS measurement. Yet, PDF uncertainties are still dominant. Moreover, the spread in central values of $\sin ^{2} \theta_{\text {eff }}^{\ell}$ measured using different PDF sets is larger than the size of the PDF uncertainties for a given set. As these PDF sets share similar input data and predictions, the spread in central values would be even more significant if one took correlations into account.

To tackle this limitation, as for the $m_{W}$ measurement, improved PDF sets including more precise LHC measurements, better predictions and a better assessment of PDF uncertainties and correlations are crucial to reach the precision goal in future $\sin ^{2} \theta_{\text {eff }}^{\ell}$ measurements. Improved PDF sets can also ease combinations of $\sin ^{2} \theta_{\text {eff }}^{\ell}$ measurements as they would avoid the need for constraining 
PDF uncertainties in-situ. Such constraints can in fact be inconsistent across measurements.

\section{Recent measurements of $W$ and $Z$ boson production differential cross sections}

The ATLAS and CMS experiments have published a wealth of measurements of differential cross sections of the $W$ and $Z$ boson productions at different center-of-mass energies $(\sqrt{s}=2.76$, $5,7,8$ and $13 \mathrm{TeV}$ ) and as a function of several observables.

Thanks to the large statistics of the collected samples and the accurate knowledge of the detector performance, the precision of these measurements is significantly better than the precision of predictions. These measurements are therefore crucial inputs for the improved predictions needed for the precision goal of the $\sin ^{2} \theta_{\mathrm{eff}}^{\ell}$ and $m_{W}$ measurements. A selection of recent measurements is presented in the following.

The CMS experiment released two measurements using $Z \rightarrow \ell \ell$ events at $\sqrt{s}=13 \mathrm{TeV}$. The first is a measurement of the total and fiducial $Z$ boson production cross section as a function of dilepton invariant mass $m^{\ell \ell}$ in the range $15<m^{\ell \ell}<3000 \mathrm{GeV}$ with up to $2.8 \mathrm{fb}^{-1}$ of data [16]. The measured cross sections are in good agreement with theoretical calculations at QCD NNLO and EW NLO accuracy. The impact of the photon-induced contribution at high $m^{\ell \ell}$ is also investigated. It is significant when compared to the prediction uncertainties, but well within the measurement statistical uncertainty.

The second is a measurement of the $Z$ production cross section differential in dilepton transverse momentum $p_{\mathrm{T}}^{\ell \ell}$, dilepton rapidity $\left|y^{\ell \ell}\right|$ and $\phi^{*}$, which is a kinematic variable correlated to $\phi^{*} \sim p_{\mathrm{T}}^{\ell \ell} / m^{\ell \ell}$ [17]. Cross sections double-differential in $p_{\mathrm{T}}^{\ell \ell}$ and $\left|y^{\ell \ell}\right|$ are also presented. Absolute cross sections are measured in the detector fiducial volume $\left(\left|\eta_{\ell}\right|<2.4\right.$ and $\left.p_{\mathrm{T}}^{\ell}>25 \mathrm{GeV}\right)$ using $35.9 \mathrm{fb}^{-1}$ of data and compared to a range of predictions, including calculations using parton shower modelling at NLO accuracy in QCD, fixed order predictions at NNLO accuracy, and resummed predictions at NNLL accuracy. Fig. 4 shows ratios of resummed and fixed order predictions to data in bins of $p_{\mathrm{T}}^{\ell \ell}$. The resummed predictions are computed with the RESBOS [18] and the GENEVA [19] simulation packages. The fixed order predictions are computed with FEWZ [20] and with a complete NNLO calculation of $Z$ boson production in association with a jet $(Z+j)[21]$. As a reference, predictions with parton shower modelling using MADGRAPH5_AMC@NLO [22] are also shown. As expected, resummed predictions model well the low- $p_{\mathrm{T}}^{\ell \ell}$ events. Predictions with REsBos, in particular, show a very good agreement with data at $p_{\mathrm{T}}^{\ell \ell}<20 \mathrm{GeV}$. Fixed order calculations are instead able to model the high- $p_{\mathrm{T}}^{\ell \ell}$ events. The $Z+j$ prediction, in particular, shows good accuracy and small uncertainties, but theoretical uncertainties even at the level of a few percent are significantly larger than the precision of the measurements. Normalised cross sections are measured with uncertainties below $0.5 \%$ for events at $p_{\mathrm{T}}^{\ell \ell}<50 \mathrm{GeV}$, as these cross sections are not affected by the uncertainty on the integrated luminosity and only partially affected by experimental uncertainties, which are correlated across measurement bins [17].

The ATLAS experiment published measurements of the $W$ and $Z$ boson production differential cross sections at $\sqrt{s}=5$ and $8 \mathrm{TeV}$. The measurement at $8 \mathrm{TeV}$ is based on $W \rightarrow \mu v$ events corresponding to an integrated luminosity of $20.2 \mathrm{fb}^{-1}$ [23]. Differential cross sections and charge asymmetry $A_{\mu}=\left(\mathrm{d} \sigma_{W^{+}} / \mathrm{d} \eta_{\mu^{+}}-\mathrm{d} \sigma_{W^{-}} / \mathrm{d} \eta_{\mu^{-}}\right) /\left(\mathrm{d} \sigma_{W^{+}} / \mathrm{d} \eta_{\mu^{+}}+\mathrm{d} \sigma_{W^{-}} / \mathrm{d} \eta_{\mu^{-}}\right)$are measured as functions of the absolute muon pseudorapidity $\left|\eta_{\mu}\right|$ in the fiducial phase space $\left(\left|\eta_{\mu}\right|<2.4, p_{\mathrm{T}}^{\mu}>25 \mathrm{GeV}\right.$, 

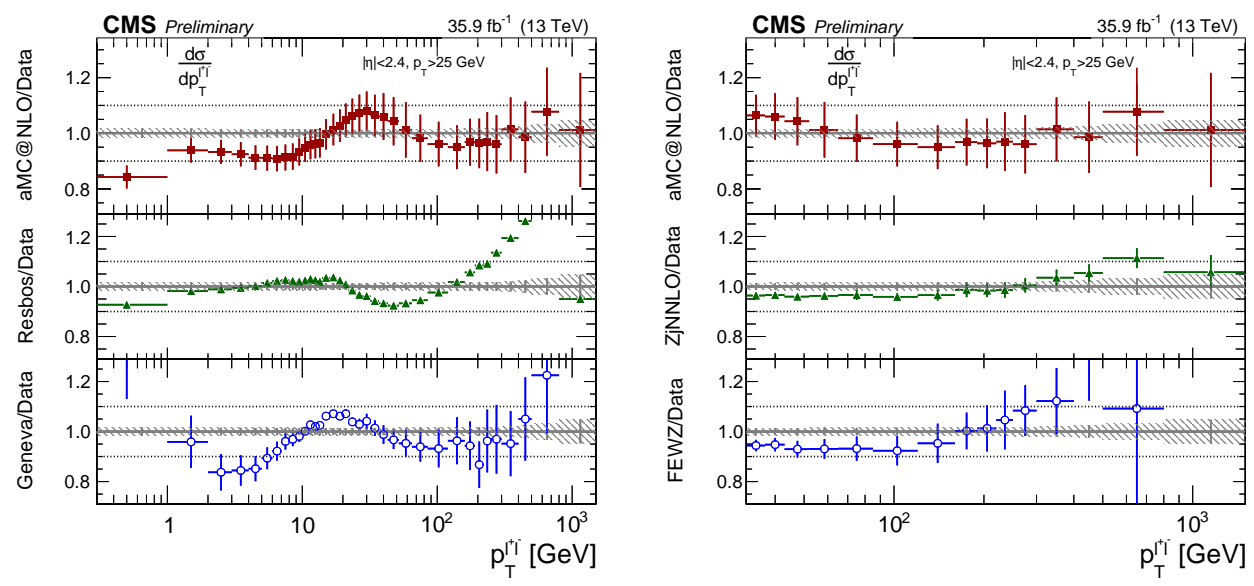

Figure 4: Ratios of predictions to data in bins of $p_{\mathrm{T}}^{\ell \ell}$. Total experimental and theoretical uncertainties are shown (only statistical uncertainties are shown for predictions with RESBOS) [17].
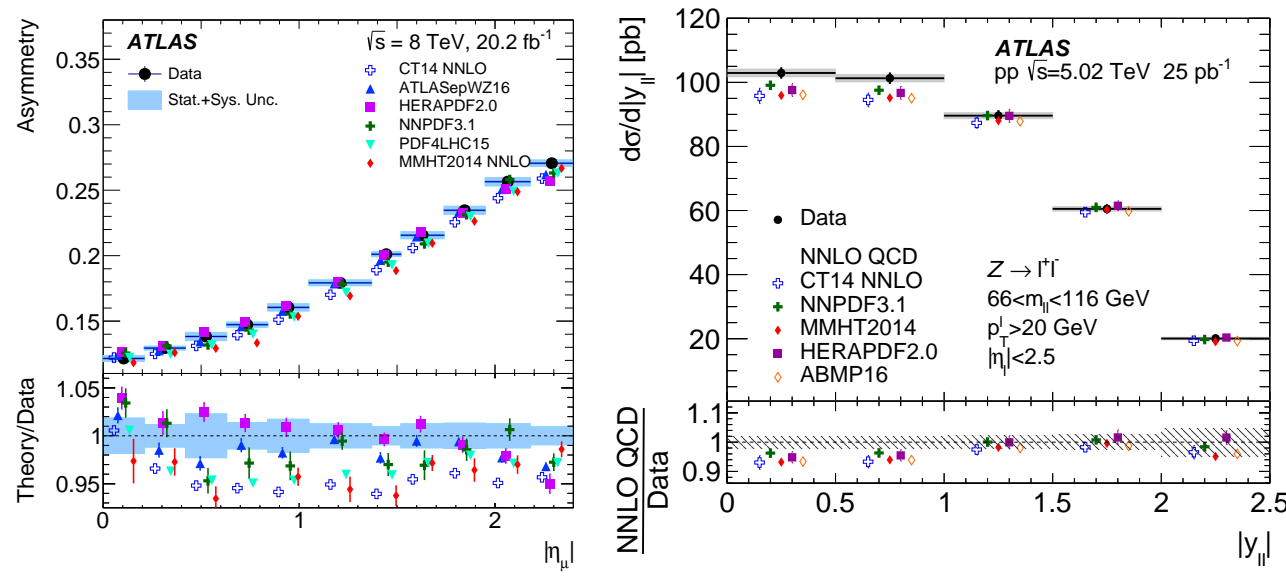

Figure 5: Comparisons of measurements of $W$ and $Z$ boson events with DYNNLO predictions with selected PDFs. Left: $W$ charge asymmetry as a function of $\left|\eta_{\mu}\right|$ [23]. Right: differential cross section for $Z$ boson production as a function of $\left|y_{\ell \ell}\right|$. The luminosity uncertainty is not included in the error bars in data [24].

$p_{\mathrm{T}}^{v}>25 \mathrm{GeV}$ and $\left.m_{\mathrm{T}}>40 \mathrm{GeV}\right)$. The precision of the cross section measurements varies between $0.8 \%$ to $1.5 \%$, excluding the $1.9 \%$ uncertainty on the integrated luminosity. The charge asymmetry is measured with an uncertainty between 0.002 and 0.003 . Results are compared with predictions based on NNLO calculations using DYNNLO [7] with various PDFs. As shown in Fig. 5 (left), the precision of the measurement is able to discriminate between predictions with different PDFs.

The measurement at $5 \mathrm{TeV}$ analyses $W \rightarrow \ell v$ and $Z \rightarrow \ell \ell$ events in $25 \mathrm{pb}^{-1}$ of $p p$ data [24]. Differential cross sections and the $W$ charge asymmetry are measured as functions of $\left|\eta_{\ell}\right|$ and $\left|y_{\ell \ell}\right|$. The precision of the measurement of 1.2-1.7\%, excluding the luminosity uncertainty (1.9\%), is dominated by uncertainties on the lepton selection efficiencies due to the small statistics of the analysed sample. Nonetheless, the precision is high enough to show $1-2 \sigma$ deviations from predictions, in particular in $Z$ boson events at low $\left|y_{\ell \ell}\right|$ (Fig. 5 right). 


\section{Summary}

Measurements of the properties of the $W$ and $Z$ bosons by the ATLAS and CMS experiments at the LHC are reaching an unprecedented precision. The two experiments are able to measure $W$ and $Z$ boson production differential cross sections at a sub-percent precision and to discriminate among predictions. This is thanks to the large samples of collected events and the accurate knowledge of the detector performance at different collision energies and pile-up levels.

The ATLAS experiment also measured $m_{W}$ and $\sin ^{2} \theta_{\text {eff }}^{\ell}$ at a precision similar to the combined measurements from the Tevatron, LEP and SLD colliders. These are key measurements to test the SM. However, their precision is still low compared to the uncertainties on the $m_{W}$ and $\sin ^{2} \theta_{\text {eff }}^{\ell}$ predicted values. The major limiting factor in the precision of these measurements are the theoretical uncertainties coming from the limited knowledge of the proton structure and the accuracy in modelling $W$ and $Z$ boson properties, like the $W$ boson transverse momentum.

The experimental and theory communities are working together towards improving the precision of future $m_{W}$ and $\sin ^{2} \theta_{\text {eff }}^{\ell}$ measurements. On the experimental side, more precise measurements with new data collected or to be collected at the LHC will be performed, in particular the measurement of the $W$ boson transverse momentum with low pile-up data. On the theoretical side, improved predictions and PDFs will be developed based on what is being learned from the high-precision LHC measurements.

To reach the experimental precision goal, efforts from both communities are needed in better assessing theoretical uncertainties and correlations, in investigating per-cent level perturbative and non-perturbative effects, and in developing frameworks to combine $\sin ^{2} \theta_{\text {eff }}^{\ell}$ and $m_{W}$ measurements.

\section{References}

[1] J. Haller et al., Eur. Phys. J. C 78 (2018) 675.

[2] ATLAS Collaboration, 2008 JINST 3 S08003.

[3] CMS Collaboration, 2008 JINST 3 S08004.

[4] ATLAS Collaboration, Eur. Phys. J. C 78 (2018) 110.

[5] P. Nason, JHEP 11 (2004) 040; S. Frixione et al., JHEP 11 (2007) 070; S. Alioli et al., JHEP 06 (2010) 043.

[6] T. Sjöstrand et al., JHEP 05 (2006) 026; T. Sjöstrand et al., Comput. Phys. Commun. 178 (2008) 852.

[7] S. Catani et al., Phys. Rev. Lett. 103 (2009) 082001.

[8] ATLAS Collaboration, JHEP 09 (2014) 145.

[9] ATLAS Collaboration, ATL-PHYS-PUB-2018-004, https://cds.cern.ch/record/2310738.

[10] ATLAS Collaboration, ATL-PHYS-PUB-2017-021, http://cdsweb.cern.ch/record/2298152.

[11] ATLAS Collaboration, Eur. Phys. J. C 77 (2017) 466.

[12] ALEPH and DELPHI and L3 and OPAL and SLD Collaborations and LEP Electroweak Working Group and SLD Electroweak Group and SLD Heavy Flavour Group, Phys. Rep. 427 (2006) 257.

[13] ATLAS Collaboration, ATLAS-CONF-2018-037, https://cds.cern.ch/record/2630340. 
[14] C. Collins and D. E. Soper, Phys. Rev. D 16 (1977) 2219.

[15] CMS Collaboration, Eur. Phys. J. C 78 (2018) 701.

[16] CMS Collaboration, https://arxiv.org/abs/1812.10529.

[17] CMS Collaboration, CMS-PAS-SMP-17-010, https://cds.cern.ch/record/2675022.

[18] G. A. Ladinsky and C. P. Yuan, Phys. Rev. D 50 (1994) R4239; C. Balazs and C. P. Yuan, Phys. Rev. D 56 (1997) 5558; F. Landry et al., Phys. Rev. D 67 (2003) 073016.

[19] S. Alioli et al., Phys. Rev. D 92 (2015), 094020.

[20] K. Melnikov and F. Petriello, Phys. Rev. Lett. 96 (2006) 231803; R. Gavin et al., Comput. Phys. Commun. 182 (2011) 2388; R. Gavin et al., Comput. Phys. Commun. 184 (2013) 208; Y. Li and F. Petriello, Phys. Rev. D 86 (2012) 094034.

[21] R. Boughezal et al., Phys. Rev. Lett. 116 (2016), 152001; R. Boughezal et al., Phys. Rev. Lett. 115 (2015), 062002.

[22] J. Alwall et al., JHEP 07 (2014) 079.

[23] ATLAS Collaboration, https://arxiv.org/abs/1904.05631.

[24] ATLAS Collaboration, Eur. Phys. J. C 79 (2019) 128. 